\section{Consumo de leite materno e fatores associados em crianças menores de dois anos: Pesquisa Nacional de Saúde, 2013}

\author{
Maternal breastfeeding and associated factors in \\ children under two years: the Brazilian National \\ Health Survey, 2013
}

\section{Consumo de leche materna y factores asociados en bebés menores de dos años: Encuesta Nacional de Salud, 2013}

Thaynã Ramos Flores 1 Bruno Pereira Nunes 2 Rosália Garcia Neves 1 Andrea T. Wendt 1

Caroline dos Santos Costa 1 Fernando C. Wehrmeister 1 Andréa Dâmaso Bertoldi 1

doi: 10.1590/0102-311X00068816

\section{Resumo}

O objetivo foi avaliar a prevalência do consumo de leite materno e os fatores associados em crianças brasileiras com menos de dois anos de idade. Estudo transversal, de base nacional, realizado em 2013. Os desfechos foram amamentação entre crianças menores de 24 meses e amamentação exclusiva entre aquelas menores de seis meses de idade. Realizou-se análise hierárquica para fatores associados. As análises foram estratificadas por idade (0-5 meses e 29 dias; 6-11 meses e 29 dias; 12-23 meses e 29 dias de idade). A prevalência de aleitamento materno foi de 56\% para o total estudado e, segundo idade, foi de 80\% (0-5 meses e 29 dias), 62,3\% (6-11 meses e 29 dias) e 40,1\% (12-23 meses e 29 dias). Nas análises ajustadas, em todas as faixas etárias, a maior prevalência de ingestão de leite materno esteve associada ao menor número de alimentos lácteos consumidos. Entre crianças de 6-11 meses e 29 dias, residir na Região Norte, ter cor da pele preta e situar-se no menor quinto de posse de bens associaram-se a maior prevalência de amamentação. Naquelas entre 12-23 meses e 29 dias, maior prevalência de consumo de leite materno foi associado à cor da pele preta, consumo de líquidos e de alimentos saudáveis, residência na zona urbana, maior escolaridade do chefe da família e posse de maior número de bens. A prevalência de aleitamento exclusivo foi de $20,6 \%$, sendo maior na Região Sul, nos maiores níveis de escolaridade do chefe da família e naquelas crianças com maior número de bens. As prevalências de amamentação e amamentação exclusiva nas crianças brasileiras menores de dois anos podem ser consideradas baixas. As politicas já existentes para aumentar as prevalências de consumo de leite materno, em qualquer faixa etária, devem ser reforçadas.

Leite Humano; Amamentação; Lactente; Consumo Alimentar
Correspondência

T. R. Flores

Programa de Pós-graduação em Epidemiologia, Universidade Federal de Pelotas.

Rua Marechal Deodoro 1160, Pelotas, RS 96020-220, Brasil. thaynaramosflores@yahoo.com.br

1 Programa de Pós-graduação em Epidemiologia, Universidade Federal de Pelotas, Pelotas, Brasil.

2 Faculdade de Enfermagem, Universidade Federal de Pelotas, Pelotas, Brasil. 


\section{Introdução}

O leite materno é considerado o melhor alimento para a criança, protegendo-a contra doenças infecciosas 1 , crônicas e agudas, além de contribuir para o adequado estado emocional e psicológico da mãe e do bebê por meio da amamentação 2,3 . A proteção que o leite materno traz para o lactente tem como consequência menores custos para os sistemas de saúde, sobretudo na redução de hospitalizações infantis 4 , além de acarretar benefícios em longo prazo, como o capital humano, uma vez que crianças com maior tempo de amamentação tendem a ter maiores níveis de renda e inteligência 5 .

Evidências sugerem que idealmente o tempo para o aleitamento materno exclusivo seria até os seis meses de idade da criança 2,6. No entanto, sabe-se que ainda existem controvérsias sobre o tempo ideal da amamentação exclusiva 3 . Em uma revisão sistemática foi verificado que crianças amamentadas exclusivamente até o sexto mês de vida não apresentaram déficits de ganho de peso e de altura na infância 4,6. Ainda, mesmo que após os seis meses de idade somente o leite materno não seja suficiente para suprir as necessidades nutricionais da criança e garantir o crescimento adequado, a amamentação não deve ser necessariamente descontinuada por permanecer fonte de proteína e outros nutrientes, como a vitamina A e ferro, por ser um alimento de fácil digestão para oferecer quando a criança perde o apetite naturalmente ou por causa de alguma enfermidade, sendo muito importante também para garantia de imunidade prolongada 2,7. Além do mais, a amamentação é um processo afetivo que envolve a mãe e a criança 2,6 .

Com relação à introdução de alimentos, uma importante preocupação é quando ela ocorre antes do sexto mês de vida, podendo resultar em infecções gastrointestinais e respiratórias, pois a introdução de alimentos complementares precocemente tem como consequência uma menor ingestão de leite materno, levando a criança a não receber os nutrientes necessários para seu desenvolvimento 8 . Ainda, além da faixa etária, os cuidados com a introdução de alimentos devem levar em conta aspectos como as necessidades energéticas, a variedade, o equilíbrio entre micro a macronutrientes na alimentação para garantir o crescimento adequado 7 .

Mesmo com a indicação de continuidade da amamentação após a introdução de outros alimentos, a adesão a essa prática em tal fase é baixa por diversos motivos 9 , entre eles, o retorno da mãe ao mercado de trabalho com consequente inserção da criança na escola ou até mesmo ter de contar com os cuidados de outra pessoa 7,10,11, e ainda, por crenças de que a amamentação possa atrapalhar a introdução de novos alimentos 2 . Pode ocorrer a falta de suporte ou incentivo às mães por parte dos serviços de saúde para garantia da amamentação exclusiva e da sua continuidade em conjunto com outros alimentos 4 .

Embora ao longo dos últimos anos tenham ocorrido melhorias, no âmbito da saúde pública, para aumento da prevalência de amamentação 12,13, ressalta-se a importância da janela de oportunidades. Essa fase, também conhecida como o período dos primeiros "1.000 dias”, que envolve desde a gestação até os 24 meses do bebê, quando tomados os cuidados necessários, garante uma série de aspectos que contribuem para a adequada saúde materno-infantil ${ }^{14}$. Dentre os cuidados com a gestação e com o bebê, que esse período requer, está a amamentação 7,14 .

Existe uma vasta literatura sobre os benefícios a curto e longo prazo da amamentação 12,15. No entanto, as últimas estimativas da prevalência da amamentação e da amamentação exclusiva baseadas em dados com representatividade nacional foram produzidas na Pesquisa Nacional de Demografia e Saúde (PNDS) de 2006 16, e as últimas estimativas produzidas para as capitais e Distrito Federal datam de 2008 (II Pesquisa de Prevalência do Aleitamento Materno) 17. Dada a importância da amamentação para a saúde da mulher e da criança 2,14,18,19,20, é oportuno atualizar essas estimativas com base em estudos mais recentes. Nesse sentido, o presente estudo tem como objetivos atualizar as prevalências de aleitamento materno entre crianças brasileiras menores de 24 meses e de aleitamento materno exclusivo entre aquelas com idade inferior a seis meses e identificar os fatores associados. 


\section{Metodologia}

Estudo transversal, de base populacional, utilizando dados da Pesquisa Nacional de Saúde (PNS) realizada em 2013, no Brasil. A pesquisa foi realizada por uma parceria entre o Instituto Brasileiro de Geografia e Estatística (IBGE) e o Ministério da Saúde. A amostra é representativa de moradores de domicílios permanentes, localizados em zona urbana ou rural, das cinco grandes regiões geográficas, 27 Unidades da Federação (UF), capitais e demais municípios. A seleção da amostra da PNS foi realizada em três etapas. Primeiro, foram selecionados os setores censitários; em seguida, os domicílios; e, por último, os indivíduos com 18 anos ou mais. A coleta dos dados, por meio de computadores de mão (personal digital assistance - PDA), foi realizada por entrevistadores treinados. Neste estudo, foram as mães ou responsáveis que responderam às questões referentes aos menores de dois anos. Maior detalhamento encontra-se no artigo metodológico da PNS 21.

Os desfechos do presente estudo foram a aleitamento materno (AM) entre crianças menores de dois anos de idade e o aleitamento materno exclusivo (AME) entre crianças menores de seis (0-5 meses e 29 dias) meses de idade. Os dados de consumo de leite materno e de outros alimentos se referiam ao dia anterior à entrevista e foram coletados com uso de questionário fechado 22 que trazia a seguinte pergunta: "Você pode me dizer quais destes alimentos <a criança> tomou ou comeu desde ontem de manhã até hoje de manhã?”. Além do leite materno, diversos alimentos foram listados. Para a construção da variável AME, foi considerado o consumo somente de leite materno, excluindo-se qualquer um dos outros alimentos sólidos ou líquidos 2,7.

Os demais alimentos foram utilizados como variáveis independentes e classificados da seguinte forma: saudáveis (frutas, verduras e legumes, feijão, arroz, carne/ovos, batata/mandioca e cereais) 2,7, lácteos (outro leite que não o materno e mingau) 2,7, não saudáveis (refrigerantes, sucos artificiais, bolachas e doces) 2,7 e os líquidos não nutritivos, ou seja, sem valor nutricional (água e chás) 2,7, todos utilizando o mesmo questionamento do desfecho. Foi construído um escore com base no somatório dos alimentos consumidos no dia anterior à entrevista em cada grupo (saudáveis, não saudáveis, lácteos e líquidos não nutritivos). Embora os alimentos lácteos e líquidos sejam também considerados saudáveis 2,7 , foram separados a fim de identificar a introdução em cada faixa etária. $\mathrm{O}$ termo "saudável" refere-se à forma de processamento dos alimentos 2,6,7, sem considerar o momento de sua introdução.

Como variáveis de ajuste, além dos alimentos, foram incluídas as seguintes: sexo da criança (masculino/feminino), cor da pele da criança (branca, preta, parda), escolaridade do chefe da família (sem instrução; Ensino Fundamental; Ensino Médio; Ensino Superior), posse de bens, que foi operacionalizada pela maioria dos itens disponíveis no questionário da PNS (banheiro dentro de casa, automóvel, motocicleta, geladeira, máquina de lavar roupas, aparelho de DVD, televisão, telefone fixo, computador e micro-ondas). Esses foram os itens com possibilidade de criação de escore conforme os critérios da Associação Brasileira de Empresas de Pesquisa (ABEP. Critério de Classificação Econômica Brasil. http://www.abep.org, acessado em 22/Mar/2016) de 2016, zona de moradia (urbana; rural), região geográfica (Norte, Nordeste, Centro-oeste, Sudeste, Sul), presença de plano de saúde (não/sim) e cobertura pela Estratégia Saúde da Família - ESF (não/sim).

O banco de dados foi obtido na página eletrônica do IBGE (http://www.ibge.gov.br/home) e as análises foram conduzidas no programa Stata 12.1 (StataCorp LP, College Station, Estados Unidos). Inicialmente, realizou-se a descrição da amostra de menores de dois anos de idade da PNS. Após, foi realizada descrição dos desfechos (AM e AME) segundo as variáveis independentes, obtendo-se suas prevalências e seus respectivos intervalos de 95\% de confiança (IC95\%). Para as análises bruta e ajustada para cada um dos desfechos, utilizou-se a regressão de Poisson 17, obtendo-se as razões de prevalência (RP), IC95\% e valor de p. As análises ajustadas foram realizadas tendo como base um modelo hierarquizado em que as variáveis demográficas, socioeconômicas, regionais, posse de plano de saúde e cobertura pela ESF se localizaram no primeiro nível e o consumo dos alimentos e líquidos no segundo nível. Foram mantidas em cada um dos níveis as variáveis que apresentaram um valor de $\mathrm{p}<0,20$, controlando para possíveis confundidores do mesmo nível e de níveis superiores. Foram consideradas estatisticamente significativas as associações que apresentaram valor de $\mathrm{p}<0,05$. Todas as análises foram realizadas pelo comando svy para considerar o efeito de delineamento amostral do estudo e estratificadas de acordo com a idade da criança sendo de 0-5 meses e 29 dias; 6-11 meses e 
29 dias; 12-23 meses e 29 dias de idade, conforme preconizado pela Organização Mundial da Saúde (OMS) e Ministério da Saúde 2,6,7,14,23.

O projeto foi submetido à Comissão Nacional de Ética em Pesquisa e aprovado sob o número de protocolo 10853812.7.0000.0008. Todos os participantes assinaram o Termo de Consentimento Livre e Esclarecido, garantindo o sigilo dos dados.

\section{Resultados}

A amostra total da PNS foi de 5.458 crianças, porém para este estudo a amostra foi de 5.044 crianças devido à disponibilidade de informações para os desfechos avaliados entre as crianças menores de 24 meses de idade. Aproximadamente 50\% da amostra eram compostos por crianças entre 13-23 meses de idade e cerca de um terço pertencia à Região Norte. Houve leve predomínio do sexo masculino $(50,2 \%)$ e pouco mais da metade era de cor da pele parda (50,9\%). Quanto à escolaridade do chefe da família, houve predomínio do nível de Ensino Fundamental completo (36\%); em relação à posse de bens, $27,3 \%$ das crianças pertenciam ao $2^{\circ}$ quinto da distribuição. A maioria das crianças residia na zona urbana $(79,1 \%)$ e a maior proporção pertencia à Região Norte (30,2\%), não tinha plano de saúde (75,9\%) e suas famílias estavam cobertas pela ESF (66\%). Aproximadamente um quarto (26,1\%) das crianças havia consumido seis alimentos saudáveis, pouco mais de um terço delas não havia consumido alimentos considerados não saudáveis (36,8\%), 40,7\% haviam ingerido dois alimentos lácteos e cerca de $75 \%$ haviam consumido pelo menos um líquido não nutritivo no dia anterior à entrevista (Tabela 1).

A prevalência de AME, entre os menores de seis meses de idade, foi de 20,6\% (IC95\%: 18,5; 22,7) com maior proporção de AME nas crianças pertencentes à Região Sul (29,4\%; IC95\%: 22,1; 36,7); naquelas que pertenciam ao quinto mais alto de posse de bens (30,5\%; IC95\%: 24,2; 36,8) (Tabela 2). Após os ajustes, as associações de AME com região e com posse de bens mantiveram-se na mesma direção e estatisticamente significativas (Tabela 3).

A prevalência de AM entre os menores de dois anos de idade foi de 56\% (IC95\%: 54,6; 57,4). Quando estratificada por faixa etária, a prevalência de amamentação foi de 80\% (IC95\%: 77,9; 82,2), 62,3\% (IC95\%: 59,6; 65,0) e de 40,2\% (IC95\%: 38,0; 42,1) entre crianças de 0-5 meses e 29 dias, 6-11 meses e 29 dias e 12-23 meses e 29 dias de idade, respectivamente. Nas crianças de 0-5 meses e 29 dias estiveram estatisticamente associadas ao AM a zona de moradia, região, posse de bens, posse de plano de saúde, consumo de alimentos considerados saudáveis e não saudáveis, alimentos lácteos e líquidos. Entre as crianças de 6-11 meses e 29 dias, observou-se associação do AM com cor da pele, região, posse de bens, consumo de alimentos considerados saudáveis e não saudáveis, alimentos lácteos e líquidos. Por fim, nas crianças de 12-23 meses e 29 dias de idade, estiveram estatisticamente associadas ao AM a cor da pele, escolaridade do chefe da família, região, posse de bens, posse de plano de saúde, consumo de alimentos considerados saudáveis e não saudáveis e de alimentos lácteos (Tabela 2).

$\mathrm{Na}$ análise ajustada, permaneceram associadas ao aleitamento materno dos 0-5 meses e 29 dias de idade apenas o consumo de pelo menos um alimento lácteo, dos 6-11 meses e 29 dias a cor da pele preta, posse de bens e região; dos 12-23 meses e 29 dias de idade, permaneceu a cor da pele, escolaridade do chefe da família, posse de bens, zona de residência, consumo de alimentos saudáveis, consumo de pelo menos três alimentos não saudáveis, consumo de lácteos e de líquidos. Assim, observou-se que para a continuidade do AM (6-23 meses e 29 dias) houve menor prevalência de crianças amamentadas entre aquelas cujos chefes da família possuíam maior nível de escolaridade (12-23 meses) e entre às pertencentes ao quinto mais alto de posse de bens (6-11 e 12-23 meses). Verificou-se, também, maior prevalência de continuidade de AM entre as crianças de cor da pele preta (6-11 e 12-23 meses) e parda (12-23 meses), pertencentes à zona urbana (12-23 meses) e à Região Norte (6-11 meses). Com relação ao consumo de outros alimentos ou bebidas, encontrou-se menor prevalência de crianças consumindo leite materno de acordo com o maior número de alimentos lácteos ingeridos em todas as faixas de idade, entretanto para continuidade de AM foi identificada menor prevalência de consumo entre as crianças que ingeriram cinco e seis alimentos saudáveis e até três não saudáveis (12-23 meses). Quanto aos líquidos não nutritivos, foi observada maior prevalência de AM entre as crianças que consumiram dois líquidos (12-23 meses) (Tabela 4). 


\section{Tabela 1}

Descrição das características da amostra de crianças menores de dois anos de idade. Pesquisa Nacional de Saúde, Brasil, 2013 (N = 5.044).

\begin{tabular}{|c|c|c|c|c|}
\hline Variáveis & $\begin{array}{c}0-5 \text { meses e } \\
29 \text { dias de idade } \\
n(\%) *\end{array}$ & $\begin{array}{c}\text { 6-11 meses e } \\
29 \text { dias de idade } \\
n(\%) *\end{array}$ & $\begin{array}{c}12-23 \text { meses e } \\
29 \text { dias de idade } \\
n(\%) *\end{array}$ & $\begin{array}{c}\text { Total da amostra } \\
\text { n (\%) * }\end{array}$ \\
\hline \multicolumn{5}{|l|}{ Sexo } \\
\hline Masculino & $703(50,0)$ & $620(48,6)$ & $1.209(51,2)$ & $2.532(50,2)$ \\
\hline Feminino & $704(50,0)$ & $655(51,4)$ & $1.153(48,8)$ & $2.512(49,8)$ \\
\hline \multicolumn{5}{|l|}{ Cor da pele ** } \\
\hline Branca & $633(45,6)$ & $535(42,3)$ & $981(42,1)$ & $2.149(42,5)$ \\
\hline Preta & $66(4,8)$ & $73(5,8)$ & $119(5,1)$ & $258(5,3)$ \\
\hline Parda & $688(49,6)$ & $658(51,9)$ & $1.233(52,8)$ & $2.579(50,9)$ \\
\hline \multicolumn{5}{|l|}{$\begin{array}{l}\text { Escolaridade chefe da } \\
\text { família }\end{array}$} \\
\hline Sem instrução & $209(14,8)$ & $167(13,1)$ & $353(14,9)$ & $729(14,4)$ \\
\hline Ensino Fundamental & $508(35,9)$ & $451(35,4)$ & $874(37,0)$ & $1.833(36,3)$ \\
\hline Ensino Médio & $474(33,5)$ & $441(34,6)$ & $795(33,6)$ & $1.710(33,9)$ \\
\hline Ensino Superior & $223(15,8)$ & $214(16,8)$ & $343(14,5)$ & $780(15,4)$ \\
\hline \multicolumn{5}{|l|}{ Posse de bens } \\
\hline 1 o quinto (mais pobre) & $334(23,7)$ & $294(23,0)$ & $594(25,1)$ & $1.222(24,2)$ \\
\hline 20 quinto & $391(27,8)$ & $358(28,1)$ & $630(26,7)$ & $1.379(27,3)$ \\
\hline 3o quinto & $251(17,8)$ & $213(16,7)$ & $439(18,6)$ & $903(17,9)$ \\
\hline 4o quinto & $227(16,1)$ & $222(17,4)$ & $379(16,0)$ & $828(16,4)$ \\
\hline 5o quinto (mais rico) & $204(14,5)$ & $188(14,8)$ & $320(13,6)$ & $712(14,1)$ \\
\hline \multicolumn{5}{|l|}{ Zona de residência } \\
\hline Rural & $285(20,3)$ & $242(19,0)$ & $526(22,3)$ & $1.053(20,9)$ \\
\hline Urbana & $1.122(79,7)$ & $1.033(81,0)$ & $1.836(77,7)$ & $3.991(79,1)$ \\
\hline \multicolumn{5}{|l|}{ Região } \\
\hline Sudeste & $258(18,3)$ & $199(15,6)$ & $429(18,2)$ & $886(17,6)$ \\
\hline Nordeste & $422(30,0)$ & $354(27,8)$ & $716(30,3)$ & $1.492(29,6)$ \\
\hline Centro-oeste & $165(11,7)$ & $173(13,6)$ & $284(12,0)$ & $622(12,3)$ \\
\hline Norte & $408(29,0)$ & $419(32,8)$ & $697(29,5)$ & $1.524(30,2)$ \\
\hline Sul & $154(11,0)$ & $130(10,2)$ & $236(10,0)$ & $520(10,3)$ \\
\hline \multicolumn{5}{|l|}{ Plano de saúde } \\
\hline Não & $1.057(75,1)$ & $961(75,4)$ & $1.807(76,5)$ & $3.825(75,8)$ \\
\hline $\operatorname{Sim}$ & $350(24,9)$ & $314(24,6)$ & $555(23,5)$ & $1.219(24,2)$ \\
\hline \multicolumn{5}{|l|}{ Cobertura pela ESF $* \star \star$} \\
\hline Não & $413(32,9)$ & $403(35,9)$ & $696(33,6)$ & $1.512(34,0)$ \\
\hline Sim & $841(67,1)$ & $720(64,1)$ & $1.375(66,4)$ & $2.936(66,0)$ \\
\hline \multicolumn{5}{|l|}{ Alimentos saudáveis \# } \\
\hline 0 & $785(56,6)$ & $93(7,4)$ & $71(3,2)$ & $949(19,5)$ \\
\hline 1 & $189(13,6)$ & $72(5,8)$ & $86(3,9)$ & $347(7,1)$ \\
\hline 2 & $77(5,6)$ & $103(8,3)$ & $144(6,5)$ & $324(6,7)$ \\
\hline 3 & $92(6,6)$ & $126(10,1)$ & $213(9,6)$ & $431(8,9)$ \\
\hline 4 & $96(6,9)$ & $198(15,9)$ & $375(16,9)$ & $669(13,8)$ \\
\hline 5 & $78(5,6)$ & $255(20,4)$ & $537(24,2)$ & $870(17,9)$ \\
\hline 6 & $71(5,1)$ & $402(32,2)$ & $794(35,7)$ & $1.267(26,1)$ \\
\hline \multicolumn{5}{|l|}{ Alimentos não saudáveis \# } \\
\hline 0 & $1.126(81,1)$ & $342(27,4)$ & $320(14,4)$ & $1.788(36,8)$ \\
\hline 1 & $180(13,0)$ & $516(41,3)$ & $601(27,1)$ & $1.297(26,7)$ \\
\hline 2 & $57(4,1)$ & $234(18,7)$ & $586(26,4)$ & $877(18,1)$ \\
\hline 3 & $16(1,2)$ & $103(8,3)$ & $466(21,0)$ & $585(12,0)$ \\
\hline 4 & $9(0,7)$ & $54(4,3)$ & $247(11,1)$ & $310(6,4)$ \\
\hline
\end{tabular}

(continua) 
Tabela 1 (continuação)

\begin{tabular}{|c|c|c|c|c|}
\hline Variáveis & $\begin{array}{c}0-5 \text { meses e } \\
29 \text { dias de idade } \\
n(\%) *\end{array}$ & $\begin{array}{c}\text { 6-11 meses e } \\
29 \text { dias de idade } \\
n(\%) *\end{array}$ & $\begin{array}{c}12-23 \text { meses e } \\
29 \text { dias de idade } \\
n(\%) *\end{array}$ & Total da amostra \\
\hline \multicolumn{5}{|l|}{ Lácteos } \\
\hline 0 & $588(42,4)$ & $247(19,8)$ & $261(11,8)$ & $1.096(22,6)$ \\
\hline 1 & $440(31,7)$ & $434(34,7)$ & $912(41,0)$ & $1.786(36,8)$ \\
\hline 2 & $360(25,9)$ & $568(45,5)$ & $1.047(47,2)$ & $1.975(40,7)$ \\
\hline \multicolumn{5}{|l|}{ Líquidos \# } \\
\hline 0 & $383(27,6)$ & $26(2,1)$ & $20(1,0)$ & $429(8,8)$ \\
\hline 1 & $761(54,8)$ & $986(78,9)$ & $1.836(82,7)$ & $3.583(73,8)$ \\
\hline 2 & $244(17,6)$ & $237(19,0)$ & $364(16,3)$ & $845(17,4)$ \\
\hline
\end{tabular}

ESF: Estratégia Saúde da Família.

* Percentual considerando o delineamento do estudo: svy;

** Cor da pele: amarela e indígena representaram menos de 1\% da amostra;

*** Dados faltantes para 632 crianças;

\# Escore de alimentos consumidos.

\section{Tabela 2}

Prevalência de aleitamento materno exclusivo e de consumo de leite materno de acordo com as variáveis independentes e com a faixa etária de crianças menores de dois anos de idade. Pesquisa Nacional de Saúde, Brasil, 2013 (N = 5.044).

\begin{tabular}{|c|c|c|c|c|}
\hline \multirow[t]{3}{*}{ Variáveis } & \multirow{3}{*}{$\begin{array}{c}\text { AME } \\
\text { 0-5 meses e } 29 \text { dias de } \\
\text { idade } \\
\text { [n=1.407] } \\
\%(I C 95 \%)\end{array}$} & \multicolumn{3}{|c|}{ AM } \\
\hline & & $\begin{array}{c}0-5 \text { meses e } 29 \text { dias de } \\
\text { idade } \\
{[n=1.407]}\end{array}$ & $\begin{array}{c}\text { 6-11 meses e } 29 \text { dias de } \\
\text { idade } \\
{[n=1.275]}\end{array}$ & $\begin{array}{c}\text { 12-23 meses e } 29 \text { dias } \\
\text { de idade } \\
{[n=2.362]}\end{array}$ \\
\hline & & $\%(I C 95 \%)$ & $\%($ IC95\%) & \% (IC95\%) \\
\hline \multicolumn{5}{|l|}{ Sexo } \\
\hline Masculino & $21,3(18,3 ; 24,4)$ & $78,9(75,8 ; 82,1)$ & $59,6(55,7 ; 63,5)$ & $41,3(38,4 ; 44,1)$ \\
\hline Feminino & $19,9(16,9 ; 22,9)$ & $81,1(78,2 ; 84,0)$ & $64,8(61,1 ; 68,6)$ & $38,9(35,9 ; 41,8)$ \\
\hline \multicolumn{5}{|l|}{ Cor da pele * } \\
\hline Branca & $22,0(18,7 ; 25,3)$ & $77,5(74,2 ; 80,8)$ & $56,7(52,3 ; 61,0)$ & $33,7(30,7 ; 36,8)$ \\
\hline Preta & $16,7(7,3 ; 26,0)$ & $80,3(70,5 ; 90,1)$ & $74,7(64,1 ; 85,2)$ & $47,0(37,8 ; 56,1)$ \\
\hline Parda & $19,7(16,7 ; 22,7)$ & $82,4(79,5 ; 85,3)$ & $65,5(61,8 ; 69,2)$ & $44,2(41,3 ; 47,1)$ \\
\hline \multicolumn{5}{|c|}{ Escolaridade chefe da família } \\
\hline Sem instrução & $20,3(14,4 ; 26,2)$ & $81,2(75,8 ; 86,6)$ & $67,1(59,9 ; 74,3)$ & $45,3(39,9 ; 50,6)$ \\
\hline Ensino Fundamental & $19,1(15,6 ; 22,5)$ & $80,5(77,0 ; 84,0)$ & $64,2(59,7 ; 68,6)$ & $44,4(41,0 ; 47,7)$ \\
\hline Ensino Médio & $20,4(16,7 ; 24,0)$ & $81,3(77,7 ; 84,9)$ & $61,1(56,4 ; 65,8)$ & $39,0(35,4 ; 42,5)$ \\
\hline Ensino Superior & $24,9(19,1 ; 30,7)$ & $75,1(69,4 ; 80,9)$ & $57,4(50,8 ; 64,0)$ & $26,6(21,9 ; 31,4)$ \\
\hline \multicolumn{5}{|l|}{ Zona de residência } \\
\hline Rural & $20,8(15,8 ; 25,8)$ & $85,7(81,2 ; 90,1)$ & $66,1(60,0 ; 72,1)$ & $42,2(38,0 ; 46,5)$ \\
\hline Urbana & $20,6(18,2 ; 22,9)$ & $78,6(76,2 ; 81,1)$ & $61,4(58,4 ; 64,4)$ & $39,5(37,1 ; 41,8)$ \\
\hline \multicolumn{5}{|l|}{ Região } \\
\hline Sudeste & $21,6(16,5 ; 26,6)$ & $75,7(70,4 ; 81,0)$ & $56,1(49,1 ; 63,0)$ & $37,7(33,1 ; 42,4)$ \\
\hline Nordeste & $14,6(11,2 ; 18,0)$ & $77,5(73,4 ; 81,7)$ & $59,2(54,0 ; 64,3)$ & $41,9(38,2 ; 45,7)$ \\
\hline Centro-oeste & $22,6(16,4 ; 28,7)$ & $81,7(75,4 ; 88,0)$ & $61,6(54,1 ; 69,1)$ & $34,2(28,5 ; 39,9)$ \\
\hline Norte & $22,0(17,7 ; 26,3)$ & $85,3(81,7 ; 88,8)$ & $70,0(65,4 ; 74,6)$ & $45,9(41,9 ; 49,8)$ \\
\hline Sul & $29,8(22,4 ; 37,2)$ & $78,8(72,2 ; 85,4)$ & $56,5(47,8 ; 65,1)$ & $29,2(23,2 ; 35,2)$ \\
\hline
\end{tabular}

(continua) 
Tabela 2 (continuação)

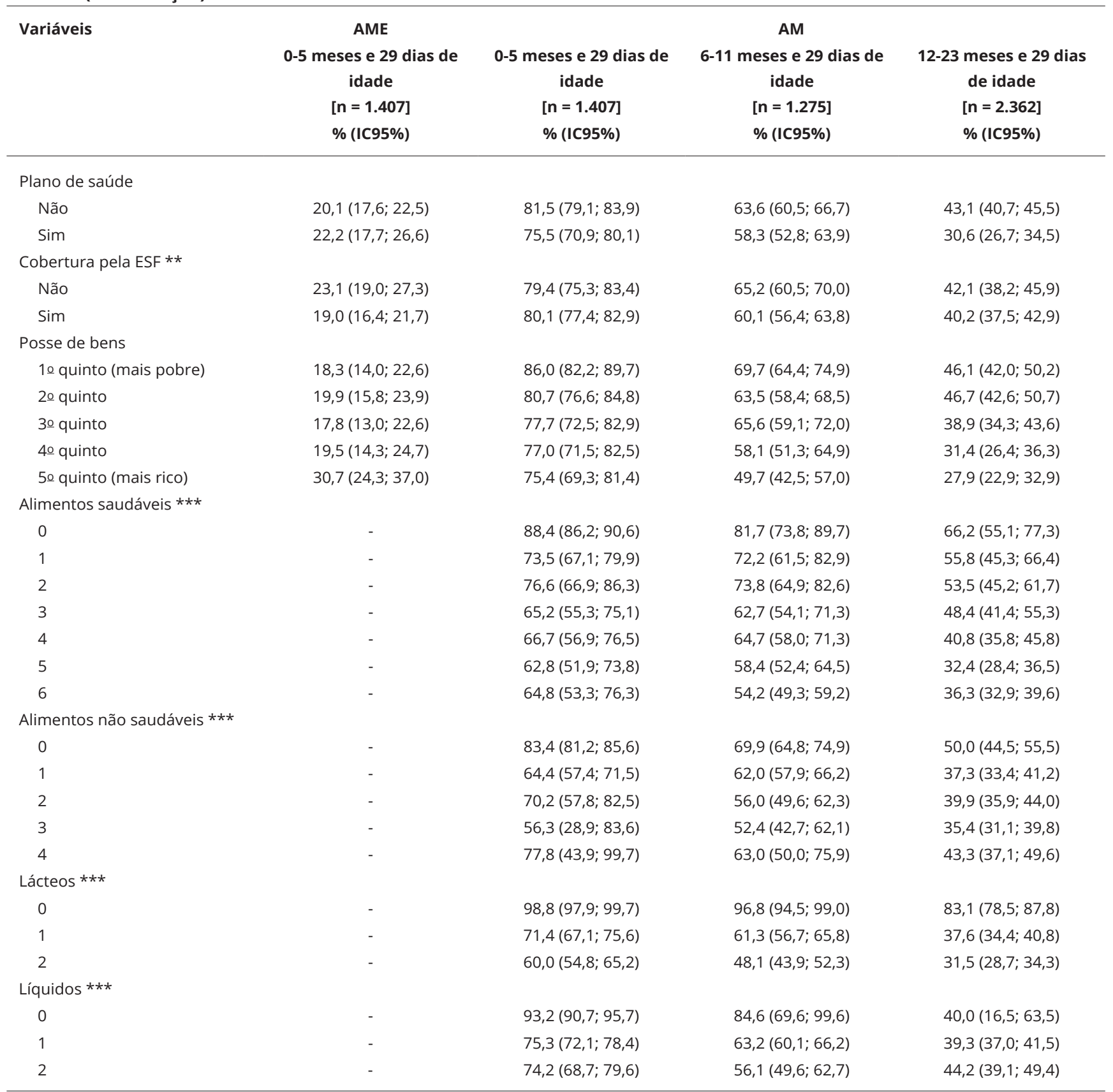

AM: Aleitamento materno; AME: Aleitamento materno exclusivo; ESF: Estratégia Saúde da Família; IC95\%: intervalo de 95\% de confiança.

* Cor da pele: amarela e indígena representaram menos de 1\% da amostra;

** Dados faltantes para 632 crianças;

*** Escore de alimentos consumidos. 


\section{Tabela 3}

Análise bruta e ajustada do aleitamento materno exclusivo (AME), em menores de seis meses de idade, segundo as variáveis independentes. Pesquisa Nacional de Saúde, Brasil, 2013 ( $n=1.407)$.

\begin{tabular}{|c|c|c|c|c|}
\hline \multirow[t]{2}{*}{ Variáveis } & \multicolumn{2}{|c|}{ Análise bruta } & \multicolumn{2}{|c|}{ Análise ajustada } \\
\hline & RP (IC95\%) & Valor de $p$ * & RP (IC95\%) & Valor de $p$ * \\
\hline Sexo & & 0,48 & & 0,61 \\
\hline Masculino & 1,00 & & 1,00 & \\
\hline Feminino & $0,93(0,75 ; 1,14)$ & & $0,95(0,77 ; 1,17)$ & \\
\hline Cor da pele ** & & 0,47 & & 0,81 \\
\hline Branca & 1,00 & & 1,00 & \\
\hline Preta & $0,76(0,43 ; 1,34)$ & & $0,84(0,48 ; 1,48)$ & \\
\hline Parda & $0,90(0,72 ; 1,11)$ & & $1,01(0,80 ; 1,28)$ & \\
\hline Escolaridade chefe da família & & $0,22 * * *$ & & $0,98 * * *$ \\
\hline Sem instrução & 1,00 & & 1,00 & \\
\hline Ensino Fundamental & $0,94(0,67 ; 1,32)$ & & $0,93(0,66 ; 1,32)$ & \\
\hline Ensino Médio & $1,01(0,72 ; 1,41)$ & & $0,95(0,67 ; 1,36)$ & \\
\hline Ensino Superior & $1,22(0,84 ; 1,77)$ & & $0,93(0,61 ; 1,41)$ & \\
\hline Zona de residência & & 0,93 & & 0,35 \\
\hline Rural & 1,00 & & 1,00 & \\
\hline Urbana & $0,99(0,76 ; 1,29)$ & & $0,87(0,65 ; 1,17)$ & \\
\hline Região & & 0,002 & & 0,01 \\
\hline Sudeste & 1,00 & & 1,00 & \\
\hline Nordeste & $0,67(0,49 ; 0,94)$ & & $0,71(0,50 ; 0,99)$ & \\
\hline Centro-oeste & $1,04(0,73 ; 1,48)$ & & $1,03(0,72 ; 1,47)$ & \\
\hline Norte & $1,01(0,75 ; 1,37)$ & & $1,09(0,79 ; 1,50)$ & \\
\hline Sul & $1,36(0,97 ; 1,91)$ & & $1,32(0,94 ; 1,86)$ & \\
\hline Plano de saúde & & 0,43 & & \\
\hline Não & 1,00 & & 1,00 & \\
\hline Sim & $1,10(0,87 ; 1,39)$ & & & \\
\hline Cobertura pela ESF \# & & 0,09 & & \\
\hline Não & 1,00 & & 1,00 & \\
\hline Sim & $0,82(0,66 ; 1,03)$ & & & \\
\hline Posse de bens & & $0,009 * * *$ & & $0,03 * * *$ \\
\hline 1ㅇ quinto (mais pobre) & 1,00 & & 1,00 & \\
\hline 2o quinto & $1,08(0,80 ; 1,48)$ & & $1,20(0,85 ; 1,69)$ & \\
\hline 3o quinto & $0,97(0,68 ; 1,38)$ & & $1,00(0,66 ; 1,50)$ & \\
\hline 4o quinto & $1,06(0,75 ; 1,51)$ & & $1,07(0,71 ; 1,63)$ & \\
\hline 5o quinto (mais rico) & $1,67(1,22 ; 2,28)$ & & $1,69(1,11 ; 2,58)$ & \\
\hline
\end{tabular}

ESF: Estratégia Saúde da Família; IC95\%: intervalo de 95\% de confiança; RP: razão de prevalência.

* Valor de p: teste de Wald para heterogeneidade;

** Cor da pele: amarela e indígena representaram menos de $1 \%$ da amostra;

$\star \star \star$ Valor de p: teste de Wald para tendência linear;

\# Dados faltantes para 632 crianças. 
Análise bruta e ajustada do consumo de leite materno de acordo com as variáveis independentes em menores de dois anos de idade. Pesquisa Nacional de Saúde, Brasil, 2013 ( $\mathrm{N}$ = 5.044).

\begin{tabular}{|c|c|c|c|c|c|c|}
\hline \multirow[t]{2}{*}{ Variáveis } & \multicolumn{2}{|c|}{$\begin{array}{l}0-5 \text { meses e } 29 \text { dias de idade } \\
{[n=1.407]}\end{array}$} & \multicolumn{2}{|c|}{$\begin{array}{l}\text { 6-11 meses e } 29 \text { dias de idade } \\
{[\quad[n=1.275]}\end{array}$} & \multicolumn{2}{|c|}{$\begin{array}{c}\text { 12-23 meses e } 29 \text { dias de idade } \\
{[n=2.362]}\end{array}$} \\
\hline & $\begin{array}{c}\text { Bruta } \\
\text { RP (IC95\%) }\end{array}$ & $\begin{array}{l}\text { Ajustada } \\
\text { RP (IC95\%) }\end{array}$ & $\begin{array}{c}\text { Bruta } \\
\text { RP (IC95\%) }\end{array}$ & $\begin{array}{l}\text { Ajustada } \\
\text { RP (IC95\%) }\end{array}$ & $\begin{array}{c}\text { Bruta } \\
\text { RP (IC95\%) }\end{array}$ & $\begin{array}{l}\text { Ajustada } \\
\text { RP (IC95\%) }\end{array}$ \\
\hline Sexo & $p=0,32$ * & $p=0,36$ * & $p=0,06$ * & $p=0,08$ * & $p=0,25$ * & $p=0,26$ * \\
\hline Masculino & 1,00 & 1,00 & 1,00 & 1,00 & 1,00 & 1,00 \\
\hline Feminino & $1,03(0,97 ; 1,08)$ & $1,03(0,97 ; 1,08)$ & $1,09(1,00 ; 1,19)$ & $1,08(0,99 ; 1,18)$ & $0,94(0,85 ; 1,04)$ & $0,94(0,85 ; 1,04)$ \\
\hline Cor da pele ** & $p=0,09$ * & $p=0,40$ * & $p<0,001$ * & $p=0,01$ * & $p<0,001$ * & $p=0,01$ * \\
\hline Branca & 1,00 & 1,00 & 1,00 & 1,00 & 1,00 & 1,00 \\
\hline Preta & $1,04(0,91 ; 1,18)$ & $1,03(0,91 ; 1,17)$ & $1,32(1,12 ; 1,54)$ & $1,28(1,09 ; 1,50)$ & $1,39(1,13 ; 1,72)$ & $1,26(1,02 ; 1,55)$ \\
\hline Parda & $1,06(1,01 ; 1,12)$ & $1,04(0,98 ; 1,10)$ & $1,16(1,05 ; 1,27)$ & $1,09(0,99 ; 1,21)$ & $1,31(1,17 ; 1,46)$ & $1,18(1,05 ; 1,32)$ \\
\hline $\begin{array}{l}\text { Escolaridade chefe da } \\
\text { família }\end{array}$ & $p=0,21 * \star \star$ & $p=0,69 * * \star$ & $p=0,03 * \star *$ & $p=0,74 * \star \star$ & $p<0,001$ *** & $p=0,02$ *** \\
\hline Sem instrução & 1,00 & 1,00 & 1,00 & 1,00 & 1,00 & 1,00 \\
\hline Ensino Fundamental & $0,99(0,92 ; 1,07)$ & $1,02(0,94 ; 1,10)$ & $0,96(0,84 ; 1,09)$ & $0,99(0,87 ; 1,12)$ & $0,98(0,85 ; 1,12)$ & $1,02(0,89 ; 1,18)$ \\
\hline Ensino Médio & $1,00(0,92 ; 1,09)$ & $1,05(0,97 ; 1,15)$ & $0,91(0,80 ; 1,04)$ & $0,96(0,84 ; 1,11)$ & $0,86(0,74 ; 1,00)$ & $0,95(0,81 ; 1,11)$ \\
\hline Ensino Superior & $0,93(0,84 ; 1,02)$ & $0,99(0,88 ; 1,11)$ & $0,86(0,73 ; 1,00)$ & $0,99(0,83 ; 1,17)$ & $0,59(0,48 ; 0,73)$ & $0,76(0,60 ; 0,96)$ \\
\hline Posse de bens & $\mathrm{p}=0,001 * * *$ & $p=0,05 * \star \star$ & $\mathrm{p}<0,001 * \star \star$ & $p=0,008 * * *$ & $\mathrm{p}<0,001 * * *$ & $p=0,001 * * *$ \\
\hline $\begin{array}{l}\text { 10 quinto (mais } \\
\text { pobre) }\end{array}$ & 1,00 & 1,00 & 1,00 & 1,00 & 1,00 & 1,00 \\
\hline 2o quinto & $0,94(0,87 ; 1,00)$ & $0,97(0,90 ; 1,05)$ & $0,92(0,82 ; 1,02)$ & $0,94(0,83 ; 1,06)$ & $1,01(0,89 ; 1,14)$ & $1,02(0,89 ; 1,17)$ \\
\hline 3o quinto & $0,90(0,83 ; 0,97)$ & $0,94(0,86 ; 1,03)$ & $0,95(0,84 ; 1,07)$ & $0,99(0,86 ; 1,14)$ & $0,84(0,72 ; 0,98)$ & $0,89(0,75 ; 1,05)$ \\
\hline 4ㅇ quinto & $0,90(0,82 ; 0,97)$ & $0,93(0,85 ; 1,02)$ & $0,84(0,73 ; 0,96)$ & $0,90(0,76 ; 1,05)$ & $0,68(0,57 ; 0,82)$ & $0,76(0,62 ; 0,93)$ \\
\hline 5o quinto (mais rico) & $0,88(0,80 ; 0,96)$ & $0,91(0,82 ; 1,01)$ & $0,71(0,60 ; 0,84)$ & $0,75(0,61 ; 0,91)$ & $0,60(0,49 ; 0,73)$ & $0,73(0,58 ; 0,93)$ \\
\hline Zona de residência & $p=0,005$ * & $p=0,19$ * & $p=0,16$ * & $p=0,60$ * & $p=0,26$ * & $p=0,03$ * \\
\hline Rural & 1,00 & 1,00 & 1,00 & 1,00 & 1,00 & 1,00 \\
\hline Urbana & $0,92(0,86 ; 0,97)$ & $0,95(0,89 ; 1,02)$ & $0,93(0,84 ; 1,03)$ & $1,03(0,92 ; 1,15)$ & $0,94(0,83 ; 1,05)$ & $1,16(1,02 ; 1,32)$ \\
\hline Região & $p=0,01$ * & $p=0,06$ * & $p=0,002$ * & $p=0,03$ * & $p<0,001$ * & $p=0,24$ * \\
\hline Sudeste & 1,00 & 1,00 & 1,00 & 1,00 & 1,00 & 1,00 \\
\hline Nordeste & $0,91(0,85 ; 0,97)$ & $0,99(0,91 ; 1,08)$ & $0,85(0,76 ; 0,94)$ & $1,00(0,86 ; 1,16)$ & $0,91(0,81 ; 1,04)$ & $1,00(0,85 ; 1,16)$ \\
\hline Centro-oeste & $0,96(0,90 ; 1,04)$ & $1,07(0,96 ; 1,18)$ & $0,88(0,77 ; 1,01)$ & $1,12(0,94 ; 1,33)$ & $0,75(0,62 ; 0,90)$ & $0,89(0,73 ; 1,09)$ \\
\hline Norte & $0,89(0,82 ; 0,96)$ & $1,09(1,00 ; 1,18)$ & $0,80(0,70 ; 0,92)$ & $1,17(1,02 ; 1,35)$ & $0,82(0,71 ; 0,96)$ & $1,03(0,88 ; 1,21)$ \\
\hline Sul & $0,92(0,84 ; 1,01)$ & $1,06(0,95 ; 1,18)$ & $0,81(0,68 ; 0,95)$ & $1,08(0,88 ; 1,32)$ & $0,64(0,51 ; 0,79)$ & $0,83(0,66 ; 1,05)$ \\
\hline Plano de saúde & $p=0,03$ * & $p=0,54$ * & $p=0,11 *$ & $p=0,40$ * & $p<0,001$ * & $p=0,29$ * \\
\hline Não & 1,00 & 1,00 & 1,00 & 1,00 & 1,00 & 1,00 \\
\hline Sim & $0,93(0,87 ; 0,99)$ & $0,97(0,89 ; 1,06)$ & $0,92(0,82 ; 1,02)$ & $1,06(0,93 ; 1,20)$ & $0,71(0,62 ; 0,82)$ & $0,93(0,78 ; 1,10)$ \\
\hline Cobertura pela ESF \# & $p=0,76$ * & $p=0,76$ * & $p=0,09$ * & $p=0,10$ * & $p=0,44$ * & $p=0,05$ * \\
\hline Não & 1,00 & 1,00 & 1,00 & 1,00 & 1,00 & 1,00 \\
\hline Sim & $1,01(0,95 ; 1,07)$ & $1,01(0,95 ; 1,07)$ & $0,92(0,84 ; 1,01)$ & $0,92(0,84 ; 1,02)$ & $0,96(0,85 ; 1,07)$ & $0,89(0,79 ; 1,00)$ \\
\hline Alimentos saudáveis \#\# & $p<0,001 * * *$ & $p=0,05 * * *$ & $\mathrm{p}<0,001 * \star \star$ & $p=0,22 * \star \star *$ & $\mathrm{p}<0,001 * \star *$ & $p=0,02 * \star \star$ \\
\hline 0 & 1,00 & 1,00 & 1,00 & 1,00 & 1,00 & 1,00 \\
\hline 1 & $0,83(0,76 ; 0,91)$ & $0,95(0,87 ; 1,03)$ & $0,88(0,74 ; 1,05)$ & $0,99(0,81 ; 1,21)$ & $0,84(0,66 ; 1,08)$ & $0,88(0,69 ; 1,12)$ \\
\hline 2 & $0,87(0,76 ; 0,98)$ & $1,02(0,90 ; 1,16)$ & $0,90(0,78 ; 1,05)$ & $1,07(0,89 ; 1,28)$ & $0,81(0,65 ; 1,01)$ & $0,99(0,79 ; 1,24)$ \\
\hline 3 & $0,74(0,63 ; 0,86)$ & $0,91(0,78 ; 1,07)$ & $0,77(0,65 ; 0,91)$ & $0,97(0,81 ; 1,17)$ & $0,73(0,59 ; 0,91)$ & $0,90(0,72 ; 1,13)$ \\
\hline 4 & $0,75(0,65 ; 0,87)$ & $0,93(0,79 ; 1,08)$ & $0,79(0,69 ; 0,91)$ & $1,05(0,89 ; 1,25)$ & $0,62(0,50 ; 0,75)$ & $0,80(0,64 ; 1,00)$ \\
\hline 5 & $0,71(0,60 ; 0,84)$ & $0,91(0,77 ; 1,07)$ & $0,72(0,62 ; 0,82)$ & $0,93(0,78 ; 1,10)$ & $0,49(0,40 ; 0,60)$ & $0,70(0,56 ; 0,87)$ \\
\hline 6 & $0,73(0,62 ; 0,87)$ & $0,88(0,73 ; 1,07)$ & $0,66(0,58 ; 0,76)$ & $0,93(0,78 ; 1,10)$ & $0,55(0,45 ; 0,66)$ & $0,78(0,63 ; 0,97)$ \\
\hline
\end{tabular}

(continua) 
Tabela 4 (continuação)

\begin{tabular}{|c|c|c|c|c|c|c|}
\hline \multirow[t]{3}{*}{ Variáveis } & \multicolumn{2}{|c|}{$\begin{array}{c}0-5 \text { meses e } 29 \text { dias de idade } \\
\text { [n }=1.407]\end{array}$} & \multicolumn{2}{|c|}{$\begin{array}{l}\text { 6-11 meses e } 29 \text { dias de idade } \\
\text { [n }=1.275]\end{array}$} & \multicolumn{2}{|c|}{$\begin{array}{l}12-23 \text { meses e } 29 \text { dias de idade } \\
{[\text { [n }=2.362]}\end{array}$} \\
\hline & Bruta & Ajustada & Bruta & Ajustada & Bruta & Ajustada \\
\hline & RP (IC95\%) & RP (IC95\%) & RP (IC95\%) & RP (IC95\%) & RP (IC95\%) & RP (IC95\%) \\
\hline Alimentos não & $p<0,001 * \star \star *$ & $\mathrm{p}=0,93 * \star \star *$ & $\mathrm{p}=0,002 * \star *$ & $p=0,12 \star \star \star *$ & $\mathrm{p}=0,07 * \star \star$ & $p=0,79 * \star \star$ \\
\hline \multicolumn{7}{|l|}{ saudáveis \#\# } \\
\hline 0 & 1,00 & 1,00 & 1,00 & 1,00 & 1,00 & 1,00 \\
\hline 1 & $0,77(0,69 ; 0,86)$ & $0,93(0,83 ; 1,05)$ & $0,89(0,81 ; 0,98)$ & $0,99(0,89 ; 1,09)$ & $0,75(0,64 ; 0,87)$ & $0,89(0,75 ; 1,05)$ \\
\hline 2 & $0,84(0,71 ; 1,00)$ & $1,03(0,86 ; 1,24)$ & $0,80(0,70 ; 0,92)$ & $0,89(0,77 ; 1,02)$ & $0,80(0,69 ; 0,93)$ & $0,91(0,77 ; 1,08)$ \\
\hline 3 & $0,67(0,44 ; 1,04)$ & $0,81(0,54 ; 1,22)$ & $0,75(0,62 ; 0,91)$ & $0,85(0,69 ; 1,03)$ & $0,71(0,60 ; 0,83)$ & $0,82(0,68 ; 0,98)$ \\
\hline 4 & $0,93(0,66 ; 1,32)$ & $1,41(0,95 ; 2,09)$ & $0,90(0,73 ; 1,11)$ & $0,98(0,78 ; 1,23)$ & $0,87(0,72 ; 1,04)$ & $0,97(0,79 ; 1,20)$ \\
\hline Lácteos \#\# & $\mathrm{p}<0,001 * * *$ & $\mathrm{p}<0,001 * * *$ & $\mathrm{p}<0,001 * * *$ & $\mathrm{p}<0,001 * * *$ & $\mathrm{p}<0,001 * * *$ & $\mathrm{p}<0,001 * * *$ \\
\hline 0 & 1,00 & 1,00 & 1,00 & 1,00 & 1,00 & 1,00 \\
\hline 1 & $0,72(0,68 ; 0,77)$ & $0,74(0,70 ; 0,79)$ & $0,63(0,59 ; 0,68)$ & $0,66(0,61 ; 0,72)$ & $0,45(0,41 ; 0,50)$ & $0,51(0,46 ; 0,57)$ \\
\hline 2 & $0,61(0,56 ; 0,66)$ & $0,63(0,57 ; 0,69)$ & $0,50(0,45 ; 0,54)$ & $0,50(0,45 ; 0,56)$ & $0,38(0,34 ; 0,42)$ & $0,42(0,37 ; 0,47)$ \\
\hline Líquidos \#\# & $\mathrm{p}<0,001 * * *$ & $p=0,72 * * *$ & $\mathrm{p}=0,008 * * *$ & $\mathrm{p}=0,40 * * *$ & $\mathrm{p}=0,09 * \star \star$ & $\mathrm{p}=0,002 * \star *$ \\
\hline 0 & 1,00 & 1,00 & 1,00 & 1,00 & 1,00 & 1,00 \\
\hline 1 & $0,81(0,77 ; 0,85)$ & $1,00(0,95 ; 1,05)$ & $0,75(0,63 ; 0,89)$ & $1,02(0,83 ; 1,24)$ & $0,98(0,57 ; 1,69)$ & $1,74(0,97 ; 3,13)$ \\
\hline 2 & $0,80(0,74 ; 0,86)$ & $1,03(0,95 ; 1,12)$ & $0,66(0,54 ; 0,81)$ & $0,95(0,76 ; 1,19)$ & $1,11(0,64 ; 1,92)$ & $1,97(1,08 ; 3,58)$ \\
\hline
\end{tabular}

ESF: Estratégia Saúde da Família; IC95\%: intervalo de 95\% de confiança; RP: razão de prevalência.

* Valor de p: teste de Wald para heterogeneidade;

** Cor da pele: amarela e indígena representaram menos de 1\% da amostra;

$\star \star \star$ Valor de $\mathrm{p}$ : teste de Wald para tendência linear;

\# Dados faltantes para 632 crianças;

\#\# Escore de alimentos consumidos.

\section{Discussão}

No geral, o consumo de leite materno foi inferior a 60\%. Entre as crianças de 0-5 meses e 29 dias de idade, foi de 80\%, caindo para 62,3\% dos 6-11 meses e 29 dias e 40,1\% dos 12-23 meses e 29 dias. Contudo, cabe ressaltar que entre os menores de seis meses, observou-se que a proporção de crianças que consumiram leite materno e também ingeriram pelo menos um dos alimentos e líquidos, como chá, água, outros leites e alimentos saudáveis, foi de aproximadamente $70 \%$, o que descaracteriza a amamentação exclusiva que é recomendada para essa faixa etária 2,6,7.

Em relação ao aleitamento materno exclusivo, encontrou-se prevalência de 20,5\% na faixa etária preconizada, sendo inferior ao evidenciado em outros estudos de abrangência nacional 16,17. No presente estudo, pode-se considerar a introdução alimentar precoce; aproximadamente $70 \%$ das crianças menores de seis meses idade haviam consumido pelo menos um alimento saudável, um líquido e um alimento lácteo e cerca de $60 \%$ haviam ingerido pelo menos um alimento não saudável, mostrando alta prevalência de introdução alimentar para a faixa etária. Na PNDS de 2006, também foi observada introdução alimentar precoce, sendo superior a 30\% dos 4-5 meses de idade 24 , determinando a cessação do AME. Em países de baixa, média e alta renda, os inquéritos mais recentes de cada local evidenciaram que aproximadamente 36 milhões de crianças não foram exclusivamente amamentadas, tendo prevalências de aproximadamente 60\% 13. Mesmo assim essas estimativas são superiores à encontrada pelo presente estudo.

Apesar de a prevalência de amamentação entre os menores de seis meses de idade ter aumentado em comparação a outros estudos 16,25,26,27, a sua continuidade até pelo menos dois anos de idade da criança e a exclusividade na oferta de somente leite materno até os seis meses de idade da criança ainda são um desafio. Embora haja políticas bem consolidadas voltadas à promoção do aleitamento materno exclusivo e ao aleitamento materno continuado até pelo menos os 24 meses 2,6,7, são diversos os motivos pelos quais ocorre o desmame, independentemente da faixa etária da criança. Esse pro- 
cesso deve ser avaliado com cautela, uma vez que as causas podem ser tanto maternas, que abrangem muitos fatores entre eles os aspectos sociodemográficos ou até mesmo comportamentais, ou por razões da própria criança que, no geral, ainda é a forma de desmame menos comum 9,11,14,28.

No presente estudo, evidenciou-se que, entre os menores de seis meses de idade, o alimento mais consumido foi o leite materno. Porém, investigando somente o consumo exclusivo de leite materno até os seis meses, observou-se que a prevalência foi muito baixa. Esse achado mostra que a amamentação exclusiva não está atingindo grande parte das crianças que tenham até seis meses de idade, o que não impede que essa criança tenha tido amamentação exclusiva mesmo que inferior ao que é preconizado 2,6,7. As possíveis explicações para que algumas crianças recebam amamentação exclusiva por menos tempo, não atingindo os seis meses de idade, podem ser as crenças de que somente o leite materno não seria mais suficiente para o ganho adequado de peso e, também, pela controvérsia que ainda existe sobre a idade ideal de introdução alimentar e especialmente de introdução de outros leites que não o humano ${ }^{3}$, configurando em amamentação predominante ou até mesmo levando à cessação.

Entre as crianças de 6-11 meses e 29 dias, pode-se verificar expressivo consumo de alimentos como carnes, ovos, batata, feijão, cereais, verduras, legumes e frutas, o que é o esperado de acordo com a recomendação de alimentação complementar para essa faixa etária 2,6,18, o que reduz em parte o consumo de leite materno. Mesmo que esses achados sejam esperados, por conta da faixa etária propicia para introdução de tais alimentos, cumpre ressaltar que existe uma cultura de que a partir de uma certa idade o leite materno não é mais nutritivo para a criança e, por esse motivo também, a amamentação é descontinuada 28. Os achados de Saldiva et al. 29 também apontam para uma diminuição na probabilidade de consumir leite materno conforme o aumento da idade, provavelmente em função da introdução de outros alimentos.

Algumas diferenças na prática da amamentação segundo variáveis sociodemográficas, como cor da pele da criança e econômicas, como escolaridade do chefe da família e posse de bens, foram evidenciadas. Em relação à cor da pele, nos menores de seis meses não foram encontradas diferenças no consumo de leite materno, mas dos seis meses até os menores de 24 meses de idade as crianças de cor da pele preta apresentaram prevalências mais altas de amamentação. Esse achado reflete em parte as desigualdades sociais que ainda persistem no país 30. Uma possível explicação é que as crianças de cor da pele preta provavelmente têm menor nível socioeconômico, menor acesso a alimentos e outros leites, tornando, muitas vezes, o leite materno uma das únicas fontes de nutrição, por ser uma alimentação sem custo e que demanda apenas do tempo e disponibilidade da mãe. Além disso, crianças de 12-23 meses e 29 dias de idade que pertenciam a famílias cujo chefe possuía maior escolaridade apresentaram menores prevalências de amamentação, e considerando que a escolaridade é um proxy de nível econômico, pode-se sugerir que crianças cujas famílias possuíam maior nível econômico não apresentaram continuidade da amamentação, após determinada faixa etária 13,28.

Nesse mesmo sentido, a construção do indicador de posse bens, que foi baseado nos mesmos itens da ABEP, permitiu verificar que o aleitamento materno exclusivo é mais frequente entre indivíduos do quinto mais alto de posse de bens e foi exatamente ao contrário quando avaliada a continuidade do AM, sendo menor a prevalência de crianças sendo amamentadas no maior quinto de posse de bens. Tal achado pode ser explicado pelo fato de que em países de baixa e média renda, as mulheres de maior nível econômico estão adotando a prática de amamentação exclusiva mais rapidamente do que as mulheres de menor nível, todavia a continuação da amamentação é mais comum entre as mulheres mais pobres 13 , o que também foi identificado no presente estudo.

A prevalência de consumo de alimentos considerados não saudáveis foi alta, mesmo tendo em conta as crianças menores de um ano, embora a associação com o consumo de leite materno não tenha sido estatisticamente significativa em nenhuma das faixas etárias, exceto para crianças com idade entre 12-23 meses e 29 dias, para as quais foi observada menor prevalência de amamentação entre aquelas que consumiram três alimentos não saudáveis. Esse resultado é de certa forma alarmante, pois esses alimentos devem ser evitados em qualquer faixa etária e particularmente entre crianças menores de dois anos de idade, período fundamental do desenvolvimento no qual ocorrem diversas modificações fisiológicas, como a formação das papilas gustativas, determinantes para a preferência por alguns alimentos 2,6. Entre as crianças de 12-23 meses e 29 dias de idade, observou-se maior prevalência no consumo de alimentos considerados não saudáveis, também chamados de ultraprocessados 31 , como refrigerantes, sucos artificiais, doces e bolachas, sendo semelhante ao encontrado por 
Garcia et al. ${ }^{19}$, que avaliou a alimentação complementar e o estado nutricional de crianças menores de dois anos de idade.

Além de a introdução de alimentos não saudáveis prejudicar a continuidade do aleitamento materno ${ }^{32}$, existem os prejuízos futuros para a criança, pois o consumo desses alimentos pode contribuir para o desenvolvimento de sobrepeso e obesidade e algumas doenças crônicas, tanto a curto como médio e longo prazo 7,33. Portanto, é possível que o desmame seja desencadeado pela oferta de alimentos não saudáveis, já que, ao adquirir outro paladar, a criança pode perder o interesse pelo leite materno ${ }^{7}$. Mesmo que não tenham sido encontradas diferenças estatisticamente significativas entre consumo de alimentos não saudáveis e de leite materno, a redução da oferta desses alimentos pode ser uma medida importante para incentivar maior tempo de duração de amamentação entre os menores de dois anos de idade. Contudo, seriam necessários outros estudos para avaliar especificamente essa associação.

Por mais que não tenha sido possível mensurar o tempo de amamentação, o estudo identificou que, quanto maior a faixa etária da criança, menor a prevalência de consumo de leite materno, o que pode suprimir alguns efeitos que o leite materno possui a médio e longo prazo na vida das crianças menores de dois anos de idade 14 , de acordo com as evidências existentes que indicam que o maior tempo de amamentação está associado ao menor risco de infecções e diarreia 15 , maior nível de escolaridade e renda na vida adulta 5 e menor prevalência de sobrepeso/obesidade e diabetes mellitus 33 .

Mesmo que a formulação da pergunta que se refere ao desfecho seja apenas indicativa ao dia anterior à entrevista e possa não representar o consumo habitual de leite materno da criança, tem sido amplamente utilizada em inquéritos populacionais por representar a atual prática de amamentação. A literatura tem utilizado esse período recordatório para investigar o consumo alimentar, inclusive de leite materno 23,25 e, inclusive, foi o mesmo utilizado em pesquisas prévias também de âmbito nacional 16,17. Além disso, um período maior que o utilizado neste inquérito poderia superestimar o consumo de leite materno, considerando que as mães tendem a responder o que é ideal. Salienta-se ainda a importância em ser um estudo de base nacional, que permite a generalização dos resultados às crianças brasileiras menores de dois anos de idade e inclui as zonas urbana e rural do país.

Os dados da PNS possibilitaram uma atualização sobre a temática. Logo, foi possível evidenciar que a prevalência de amamentação exclusiva, no Brasil, foi baixa, sendo muito inferior ao desejado. Ainda, neste estudo foi observado que o alimento mais consumido entre crianças menores de seis meses de idade foi o leite materno, entretanto cabe frisar que a oferta de outros alimentos e leites foi considerada alta, descaracterizando o processo de AME. Esses achados são importantes para nortear as ações de promoção da amamentação já existentes, podendo-se identificar quais os grupos mais vulneráveis à amamentação não exclusiva e, também, às menores prevalências de amamentação. Em comparação com outros alimentos e leites, a amamentação possui uma grande vantagem porque é uma medida sem custos financeiros e possui diversos benefícios para a saúde materno-infantil, ratificando a importância de sua continuidade e da promoção dessa prática em todas as oportunidades possíveis de contato com a mãe e a criança. 


\section{Colaboradores}

T. R. Flores idealizou a temática, participou da revisão bibliográfica, redação do manuscrito, análise dos dados, interpretação e montagem dos resultados. B. P. Nunes participou da montagem do banco de dados, revisão das análises e do manuscrito. $\mathrm{R}$ G. Neves revisou a bibliografia, auxiliou redigindo o manuscrito e na revisão crítica das análises e da redação do manuscrito. A. T. Wendt, C. S. Costa e A. D. Bertoldi participou da revisão crítica do manuscrito. F. C. Wehrmeister participou da montagem do banco de dados e revisão crítica do manuscrito.

\section{Agradecimentos}

Agradecemos ao Ministério da Saúde e ao Instituto Brasileiro de Geografia e Estatística (IBGE) pela realização e disponibilização dos dados da Pesquisa Nacional de Saúde de 2013 e aos participantes do estudo.

\section{Referências}

1. Duijts L, Jaddoe V, Hofman A, Moll H. Prolonged and exclusive breastfeeding reduces the risk of infectious diseases in infancy. Pediatrics 2010; 126:e18-25.

2. Departamento de Atenção Básica, Secretaria de Atenção à Saúde, Ministério da Saúde. Saúde da criança: nutrição infantil: aleitamento materno e alimentação complementar. Brasília: Ministério da Saúde; 2009. (Série A. Normas e Manuais Técnicos). (Cadernos de Atenção Básica, 23).

3. Kramer MS, Kakuma R. Optimal duration of exclusive breastfeeding. Cochrane Database Syst Rev 2002; (1):CD003517.

4. Renfrew M, Pokhrel S, Quigley M, McCormick F, Fox-Rushby J, Dodds R, et al. Preventing disease and saving resources: the potential contribution of increasing breastfeeding rates in the UK. London: United Nations Children's Fund; 2012.

5. Victora C, Horta B, Mola C, Quevedo L, Pinheiro R, Gigante D, et al. Association between breastfeeding and intelligence, educational attainment, and income at 30 years of age: a prospective birth cohort study from Brazil. Lancet Glob Health 2015; 3:e199-205.

6. World Health Organization. Report of the expert consultation on the optimal duration of exclusive breastfeeding. Geneva: World Health Organization; 2001.

7. Departamento de Atenção Básica, Secretaria de Atenção à Saúde, Ministério da Saúde. Dez passos para uma alimentação saudável: guia alimentar para crianças menores de dois anos. Um guia para o profissional da saúde na atenção básica. 2a Ed. Brasília: Ministério da Saúde; 2013.

8. Monte C, Giuliani E. Recommendations for the complementary feeding of the breastfed child. J Pediatr (Rio J.) 2004; 80(5 Suppl): 131-41.

9. França G, Brunken G, Silva S, Escuder M, Venancio S. Determinantes da amamentação no primeiro ano de vida em Cuiabá, Mato Grosso. Rev Saúde Pública 2007; 41:711-8.

10. Barbosa M, Palma D, Domene S, Taddei J, Lopez F. Fatores de risco associados ao desmame precoce e ao período de desmame em lactentes matriculados em creches. Rev Paul Pediatr 2009; 27:272-81.

11. Carrascoza K, Possobon R, Ambrosano G, Costa Júnior A, Moraes A. Determinantes do abandono do aleitamento materno exclusivo em crianças assistidas por programa interdisciplinar de promoção à amamentação. Ciênc Saúde Coletiva 2011; 16:4139-46.

12. Horta B, Bahl R, Martines J, Victora C. Evidence on the long-term effects of breastfeeding: systematic review and meta-analyses. Geneva: Wold Health Organization; 2007.

13. Victora C, Bahl R, Barros A, França G, Horton $\mathrm{S}$, Krasevec J, et al. Breastfeeding in the $21 \mathrm{st}$ century: epidemiology, mechanisms, and lifelong effect. Lancet 2016; 387:475-90. 
14. Save the Children. Nutrition in the first 1,000 days state of the World's Mothers 2012. Fairfield: Save the Children; 2012.

15. Horta B, Victora C. Short-term effects of breastfeeding: a systematic review on the benefits of breastfeeding on diarrhoea and pneumonia mortality. Geneva: Wold Health Organization; 2013.

16. Ministério da Saúde. Pesquisa Nacional de Demografia e Saúde da Criança e da Mulher PNDS 2006: dimensões do processo reprodutivo e da saúde da criança. Brasília: Ministério da Saúde; 2009. (Série G. Estatística e Informação em Saúde).

17. Departamento de Ações Programáticas e Estratégicas, Secretaria de Atenção à Saúde, Ministério da Saúde. II pesquisa de prevalência de aleitamento materno nas capitais brasileiras e Distrito Federal. Brasília: Ministério da Saúde; 2009. (Série C. Projetos, Programas e Relatórios).

18. Oliveira L, Assis A, Pinheiro S, Prado M, Barreto M. Alimentação complementar nos primeiros dois anos de vida. Rev Nutr (Campinas) 2005; 18:459-69.

19. Garcia M, Granado F, Cardoso M. Alimentação complementar e estado nutricional de crianças menores de dois anos atendidas no Programa Saúde da Família em Acrelândia, Acre, Amazônia Ocidental Brasileira. Cad Saúde Pública 2011; 27:305-16.

20. Corrêa E, Corso A, Moreira E, Kazapi I. Alimentação complementar e características maternas de crianças menores de dois anos de idade em Florianópolis (SC). Rev Paul Pediatr 2009; 27:258-64.

21. Souza-Júnior P, Freitas M, Antonaci G, Szwarcwald CL. Desenho da amostra da Pesquisa Nacional de Saúde 2013. Epidemiol Serv Saúde 2015; 24:207-16.

22. Instituto Brasileiro de Geografia e Estatística. Pesquisa Nacional de Saúde, PNS, 2013. Questionário dos moradores do domicílio. http:// www.ibge.gov.br/home/estatistica/popula cao/pns/2013/ (acessado em 17/Nov/2015).

23. World Health Organization. Indicators for assessing infant and young child feeding practices. Part I: definitions. Conclusions of a Consensus Meeting held 6-8 November 2007 in Washington D.C., USA. http://apps.who.int/iris/ bitstream/10665/43895/1/9789241596664_ eng.pdf (acessado em 20/Jul/2016).
24. Segall-Corrêa A, Marín-León L, Panigassi G, Rea M, Pérez-Escamilla R. Amamentação e alimentação infantil. In: Ministério da Saúde, organizador. Pesquisa Nacional de Demografia e Saúde da Criança e da Mulher - PNDS 2006: dimensões do processo reprodutivo e da saúde da criança. Brasília: Ministério da Saúde; 2009. p. 195-212. (Série G. Estatística e Informação em Saúde).

25. Venancio S, Escuder M, Saldiva S, Giugliani EJ. A prática do aleitamento materno nas capitais brasileiras e Distrito Federal: situação atual e avanços. J Pediatr (Rio J.) 2010; 86:317-24.

26. Albernaz E, Araújo C, Tomasi E, Mintem G, Giugliani E, Matijasevich A, et al. Influence of breastfeeding support on the tendencies of breastfeeding rates in the city of Pelotas (RS), Brazil, from 1982 to 2004. J Pediatr (Rio J.) 2008; 84:560-4.

27. Victora CG, Matijasevich A, Santos IS, Barros AJD, Horta BL, Barros FC. Breastfeeding and feeding patterns in three birth cohorts in Southern Brazil: trends and differentials. Cad Saúde Pública 2008; 24 Suppl 3:S409-16.

28. Ramos C, Almeida J. Alegações maternas para o desmame: estudo qualitativo. J Pediatr (Rio J.) 2003; 79:385-90.

29. Saldiva S, Escuder M, Mondini L, Levy R, Venancio S. Feeding habits of children aged 6 to 12 months and associated maternal factors. J Pediatr (Rio J.) 2007; 83:53-8.

30. Paim J, Travassos C, Almeida C, Bahia L, Macinko J. The Brazilian health system: history, advances, and challenges. Lancet 377:1778-97.

31. Louzada M, Mantins A, Canela D, Baraldi L, Levy R, Claro R, et al. Alimentos ultraprocessados e perfil nutricional da dieta no Brasil. Rev Saúde Pública 2015; 49:38.

32. Vieira G, Silva L, Vieira T, Almeida J, Cabral V. Hábitos alimentares de crianças menores de 1 ano amamentadas e não amamentadas. J Pediatr (Rio J.) 2004; 80:411-6.

33. Horta B, Mola C, Victora C. Long-term consequences of breastfeeding on cholesterol, obesity, systolic blood pressure and type 2 diabetes: a systematic review and meta-analysis. Acta Pædiatr 2015; 104:30-7. 


\section{Abstract}

The objective was to assess the prevalence of maternal breastfeeding and associated factors in Brazilian children under two years of age. This was a cross-sectional nationwide study in 2013. The outcomes were breastfeeding in children under 24 months and exclusive breastfeeding under six months of age. A hierarchical analysis was performed for associated factors. The analyses were stratified by age (0-5 months and 29 days; 6-11 months and 29 days; 12-23 months and 29 days of age). Prevalence of maternal breastfeeding was $56 \%$ for the total sample, and as follows according to age: $80 \%$ (0-5 months and 29 days), 62.3\% (6-11 months and 29 days), and 40.1\% (12-23 months and 29 days). In the adjusted analyses, in all the age brackets, higher prevalence of breastfeeding was associated with consumption of fewer milk products. In children from 6 months to 11 months and 29 days, living in the North of Brazil, black skin color, and the lowest quintile of household assets were associated with higher breastfeeding prevalence. In children from 12 months to 23 months and 29 days of age, higher breastfeeding prevalence was associated with black skin color, consumption of healthy liquids and foods, living in urban areas, head-of-household's higher educational level, and more household assets. Overall prevalence of exclusive breastfeeding was 20.6\% and was higher in the South, in families with head-of-household's higher educational level, and more household assets. The prevalence rates for breastfeeding and exclusive breastfeeding of Brazilian children under two years can be considered low. Existing policies to increase the prevalence rates of maternal breastfeeding at any age should be reinforced.

\section{Human Milk; Breast Feeding; Infant; Food Consumption}

\section{Resumen}

El objetivo fue evaluar la prevalencia del consumo de leche materna y los factores asociados en bebés brasileños con menos de dos años de edad. Estudio transversal, de base nacional, realizado en 2013. Los resultados fueron lactancia entre bebés menores de 24 meses y lactancia exclusiva entre aquellos menores de seis meses de edad. Se realizó un análisis jerárquico de factores asociados. Los análisis fueron estratificadas por edad (0-5 meses y 29 dias; 6-11 meses y 29 dias; 12-23 meses y 29 días de edad). la prevalencia de la lactancia materna fue de $56 \%$ para el total estudiado y, según edad, fue de un 80\% (0-5 meses y 29 dias), 62,3\% (6-11 meses y 29 dias) y 40,1\% (12-23 meses y 29 días). En los análisis ajustados, en todas las franjas de edad, la mayor prevalencia de ingesta de leche materna estuvo asociada al menor número de alimentos lácteos consumidos. Entre bebés de 6-11 meses y 29 dias, residir en la Región Norte, tener el color de piel negro y situarse en el menor franja de renta se asociaron a una mayor prevalencia de lactancia. En aquellas entre 12-23 meses y 29 dias, la mayor prevalencia de consumo de leche materna se asoció al color de piel negra, consumo de líquidos y de alimentos saludables, residencia en una zona urbana, mayor escolaridad del jefe de familia y posesión de un mayor número de bienes. La prevalencia de la lactancia exclusiva fue de un 20,6\%, siendo mayor en la Región Sur, en los mayores niveles de escolaridad del jefe de familia y en aquellos niños con un mayor número de bienes. las prevalencias de lactancia y lactancia exclusiva en los niños brasileños menores de dos años pueden ser consideradas bajas. Las politicas ya existentes para aumentar las prevalencias de consumo de leche materna, en cualquier franja de edad, deben ser reforzadas.

Leche Humana; Lactancia; Lactante; Consumo de Alimentos
Recebido em 25/Abr/2016

Versão final reapresentada em 13/Out/2016

Aprovado em 28/Nov/2016 